\title{
KINEMATICS OF NEARBY GAS AND STARS
}

Thomas Goulet and William L.H. Shuter

Department of Physics, University of British Columbia

\section{$\underline{\text { ABSTRACT }}$}

The kinematic properties of gas and stars in the Solar neighborhood are described in terms of the 1ine-of-sight component of a three-dimensional first order Taylor series expansion of the local velocity fleld. The types of object analysed are (1) $21 \mathrm{~cm}$ absorbing clouds (2) intercloud medium (3) main sequence B stars closer than $200 \mathrm{pc}$ (4) B stars of luminosity class ranging from I to IV (5) main sequence A stars (6) K-giant stars. The least squares fitting procedure used to derive the 10 coefficients describing each of the six velocity fields was essentially the same, so that a valid comparison could be made. Marked departures from circular motion are found in most cases, but the only systematic trend is a correlation between $\partial u / \partial x$ ( $u$ being the velocity component along the $x$-axis directed towards the Galactic center) and stellar spectral type, where the gas behaves like a medium 'younger' than the early type stars. Our analysis of the gas indicated that the standard plane-parallel model provided a good description for the intercloud medium, but was inadequate for the absorbing clouds. A velocity ellipsoid description of the residuals is presented for each type of object. The influence of the Gould belt on local kinematics is discussed.

\section{INTRODUCTION}

When the velocity field around the Sun is represented by a threedimensional first order Taylor series, as was done by Helfer (1961), the 1ine-of-sight component, $V_{\ell}$, takes the form:

$$
\begin{aligned}
\mathrm{V}_{\ell} & =\mathrm{K}(1)+\mathrm{K}(2) \cos \mathrm{b} \cos \ell+\mathrm{K}(3) \cos b \sin \ell+\mathrm{K}(4) \sin b \\
& +\mathrm{K}(5) \mathrm{d} \cos ^{2} \mathrm{~b} \cos ^{2} \ell+\mathrm{K}(6) \mathrm{d} \cos ^{2} \mathrm{~b} \sin ^{2} \ell+\mathrm{K}(7) \mathrm{d} \sin ^{2} b \\
& +\mathrm{K}(8) \mathrm{d} \cos ^{2} \mathrm{~b} \sin 2 \ell+\mathrm{K}(9) \mathrm{d} \sin 2 b \sin \ell+\mathrm{K}(10) \mathrm{d} \sin 2 b \cos \ell
\end{aligned}
$$

where $d$ is the distance from the Sun.

In this expression $K(1)$ is the usual ' $K$ term', $K(2)-K(4)$ give the reflex of the 3 components $\left(u_{\theta}, v_{\theta}, w_{\theta}\right)$ of the Solar motion with respect to the LSR, and the remaining coefficients represent velocity gradients as follows:

$$
\begin{aligned}
& K(5)=(\partial \mathrm{u} / \partial \mathrm{x})_{\mathrm{d}=0} \quad: \mathrm{K}(6)=(\partial \mathrm{v} / \partial \mathrm{y})_{\mathrm{d}=0}: \mathrm{K}(7)=(\partial \mathrm{w} / \partial \mathrm{z})_{\mathrm{d}=0} \\
& \mathrm{~K}(8)=\frac{1}{2}(\partial \mathrm{u} / \partial \mathrm{y}+\partial \mathrm{v} / \partial \mathrm{x})_{\mathrm{d}=0} \\
& \mathrm{~K}(9)=\frac{1}{2}(\partial \mathrm{v} / \partial \mathrm{z}+\partial \mathrm{w} / \partial \mathrm{y})_{\mathrm{d}=0} \\
& \mathrm{~K}(10)=\frac{1}{2}(\partial \mathrm{w} / \partial \mathrm{x}+\partial \mathrm{u} / \partial \mathrm{z})_{\mathrm{d}=0}
\end{aligned}
$$

where $\mathrm{x} \& \mathrm{u}$ are directed towards the Galactic center, $\mathrm{y} \& \mathrm{v}$ towards $\ell=90^{\circ}$, and $z \& w$ towards the Galactic North pole. 
The coefficients $K(5)-K(7)$ represent velocity gradients as indicated, and $K(8)-K(10)$ are velocity shear terms. In the case of pure differential circular motion about the Galactic center all these coefficients are zero except $K(8)$ which represents the usual Oort A constant.

\section{ANALYSIS}

The $21 \mathrm{~cm}$ absorption data analysed were from the Nancay $21 \mathrm{~cm}$ Absorption Survey of Crovisier et a1. (1978), and the $21 \mathrm{~cm}$ emission data representing the intercloud medium were from Henderson (1973), and were restricted to intermediate Galactic latitudes $\left(10^{\circ}<|b|<30^{\circ}\right)$.

In analysing the $21 \mathrm{~cm}$ absorption data we found that the usual planeparallel model did not properly describe the distribution of clouds. Their average distance, $\langle d\rangle$, was given much better by a relation of the form

$$
\langle\mathrm{d}\rangle=\mathrm{c}\left(\mathrm{d}_{\mathrm{min}}+\mathrm{z}_{\mathrm{o}} \operatorname{cosec}|\mathrm{b}|\right)
$$

in which $d_{m i n}$ represents a minimum distance to the clouds, and $z_{o}$ is a scale height. $C$ is a constant equal to $15 / \mathrm{A}$ which is unity if one assumes, as we $\mathrm{did}$, that the Oort constant $A=15 \mathrm{~km} \mathrm{~s}^{-1} \mathrm{kpc}^{-1}$. We restricted our analysis to Galactic latitudes with magnitude greater than $10^{\circ}$, and for this sample we found $\mathrm{d}_{\mathrm{min}}=234 \mathrm{pc}$ and $\mathrm{z}_{\mathrm{o}}=74 \mathrm{pc}$. In this and a11 subsequent cases we rejected data which in an initial trial fit gave residuals greater than 30. The values of the coefficients for all velocity fields are 1isted in Table $I$, and plots of each fleld in the Galactic midplane are shown in Figures 1 and 2 .

In the case of the $21 \mathrm{~cm}$ emission data we found $\mathrm{d}_{\min }=0, \mathrm{z}_{\mathrm{o}}=113 \mathrm{pc}$, and therefore used the standard model.

The data for all stellar groups were obtained from Sky Catalogue 2000.0. We selected stars with measured distances and 1ine-of-sight velocities, and all stars with noted peculiarities were rejected. In contrast to the case for the gas, our analysis leads to a determination of the Oort constant A for each group.

The nodal deviation, $l$, obtained when the terms $K(5)-K(10)$ are replaced by a single term of the form $A^{\prime} d \cos ^{2} b \sin 2\left(l-l_{0}\right)$ is also given in Table I for each field.

The average residual, $\sigma$, was determined for each group. We attempted a velocity ellipsoid analysis on the residuals for each group, in which we made a standard assumption that one of the axes of the ellipsoid was perpendicular to the Galactic plane. Values of $\sigma$, the ellipsold axes $\left\langle\mathrm{u}^{2}\right\rangle / \frac{1}{2},\left\langle\mathrm{v}^{2}\right\rangle^{\frac{1}{2}}$ and $\left\langle\mathrm{w}^{2}\right\rangle^{\frac{1}{2}}$, and the vertex deviation, $l_{\mathrm{v}}$, are 1isted in Table 1 . In the case of both $21 \mathrm{~cm}$ absorption and emission, we found $\left\langle w^{2}\right\rangle$ to be negative, and thus the ellipsoids could not be determined.

\section{DISCUSSION}

Here we elaborate on the numerical results presented in Table $I$.

The coefficient $K(1)$ - the ' $K$ term' - was not determined for the gas because it was highly correlated with some of the other coefficients - see e.g. Takakubo (1967). For the stellar groups it was generally not significant except for the BI-IV stars, for which it takes a positive value 
as found in several previous studies.

The coefficients $K(2)-K(4)$ giving the Solar motion are generally similar for all groups with the exception of $v_{0}=11.7 \mathrm{~km} \mathrm{~s}^{-1}$ for AV stars which, although in agreement with other studies, is significantly smaller than the value found for the rest of our objects.

Of the coefficients $K(5)-K(7)$ which represent velocity gradients, $K(5)=\partial u / \partial x$ shows a clear systematic decrease with increasing 'age' of the studied objects. It ranges from positive values for the gas to a large negative value for the $K-g i a n t s . ~ K(6)=\partial v / \partial y$ is not generally significantly different from zero, and the same applies to $K(7)=\partial w / \partial z$ in the case of the stars. In the case of the gas, $K(7)$ takes a negative value for the absorbing clouds suggesting an inflow from both Galactic poles which is consistent with the picture given by McGee and Murray (1961) based on 21 cm emission studies. Figure 3(a) shows that this inflow is matched by an outflow in the Galactic plane, and this is kinematically consistent with the suggestion made by Weaver (1974). Figure 3(b) shows the corresponding situation for BI-IV stars, and in this case an inflow is seen from latitudes $\sim \pm 60^{\circ}$ but there is no outflow. We do not regard the positive value of $\mathrm{K}(7)$ derived from $21 \mathrm{~cm}$ emission data as being astronomically significant, because Henderson did not observe polar latitudes.

$K(8)-K(10)$ represent velocity shears. Of these only $K(8)$, which corresponds to the Oort constant A, takes significant values. In all cases we find a value smaller than the standard one of $15 \mathrm{~km} \mathrm{~s}^{-1} \mathrm{kpc}^{-1}$.

The nodal deviation, $\ell_{0}$, which depends on $K(5)$ and $K(6)$, shows a systematic tendency to Increase with increasing 'age' of the studied objects. This is related to the change of $\mathrm{K}(5)$ with age. The anomalous value for the $B V$ stars may be attributed to the large positive value of $K(6)$ determined for this group.

We now consider the velocity residuals obtained from our fits, and their description in terms of velocity ellipsolds.

The average residual, $\sigma$, shows the well studied increase with age. The low value noted for the $21 \mathrm{~cm}$ emission data may be ascribed to the fact that Henderson presented a single velocity, representing an average weighted by brightness temperature, for each spectrum in his catalog. This method of averaging gives low weight to the individual peaks representative of clouds. For this reason we believe that our analysis of Henderson's data described the intercloud medium, which is known to be kinematically 'smooth'.

Our values for the velocity ellipsoid dispersion parameters obtained from stellar data generally are in good agreement with those presented by Mihalas and Binney (1981), except in the case of BI-IV stars where our value of $\left\langle w^{2}\right\rangle^{\frac{1}{2}}$ is larger than the standard one. This discrepancy is resolved when the velocity ellipsoid is determined, as is usually done, from residuals after only Solar motion terms have been subtracted.

For both sets of $21 \mathrm{~cm}$ data we were unable to determine velocity ellipsolds because, as previously mentioned, we found $\left\langle w^{2}\right\rangle$ to be negative. However, we were able to derive a velocity ellipsold for a subset of the absorption data in which we selected only those spectra that showed a single absorption line. This subset, for which the average distance was Independent of $b$ and best described by a constant equal to $250 \mathrm{pc}$, had an average velocity residual $\sigma=3.6 \mathrm{~km} \mathrm{~s}^{-1}$ which is approximately half the 
value for the complete set. The values of the velocity ellipsoid parameters were $\left\langle\mathrm{u}^{2}\right\rangle \frac{1}{2}=3.0 \mathrm{~km} \mathrm{~s}^{-1},\left\langle\mathrm{v}^{2}\right\rangle \frac{1}{2}=3.6 \mathrm{~km} \mathrm{~s}^{-1}$ and $\left\langle w^{2}\right\rangle \frac{1}{2}=4.0 \mathrm{~km} \mathrm{~s}^{-1}$. It appears that our inability to determine an ellipsoid for the complete set can be attributed to the absorption spectra with multiple features. These spectra give large velocity residuals and tend to be concentrated at low Galactic latitudes. Taken together these two factors create an artificial variation of $\sigma$ with $b$ which interferes with the determination of the velocity ellipsoid parameters. Although we were unable to treat the $21 \mathrm{~cm}$ emission data in the same way, we suspect that a similar mechanism prevented the determination of the velocity ellipsoid.

Since many previous studies have suggested that the Gould belt plays an important role in the kinematics of the Solar neighborhood, we attempted to investigate its influence on our velocity field determinations. Referring to the work of 0lano (1982) we rejected all data within his planar description of the belt between Galactic latitudes $\pm 20^{\circ}$. We then redetermined velocity field coefficients for all groups and found no significant differences. This does not preclude the possibility that the Internal kinematics of the Gould belt is atypical, but does indicate that the motions within the Gould belt do not perturb in any significant way the description of Solar neighborhood kinematics in terms of velocity fields.

\section{CONCLUDING REMARKS}

The most significant results of this work appear to be the following :

(1) Although in individual cases there exist significant departures from circular motion, these are not uniform for all groups but vary either randomly or show trends as a function of 'age'.

(2) The most noteworthy trend is for the velocity gradient $K(5)=\partial u / \partial x$ to decrease with increasing age. This is accompanied by a corresponding increase in the related parameter, the nodal deviation $l_{0}$

(3) Our analysis of $21 \mathrm{~cm}$ absorption data shows that the planeparallel model does not provide an adequate description of their spatial distribution, since there appears to be a deficit of absorbing clouds within $200 \mathrm{pc}$ of the Sun. On the other hand the $21 \mathrm{~cm}$ intercloud medium does have a stratified distribution.

(4) We find that $21 \mathrm{~cm}$ absorbing clouds appear to move towards the Sun from the Galactic poles, and away from it in the Galactic plane. Because of the larger statistical errors in the stellar data, velocity gradients of comparable magnitude would have been difficult to detect.

(5) We have not studied the internal kinematics of the Gould belt. However, we can assert that possible anomalous kinematics within the Gould belt does not appear to affect the velocity fields we determined.

A more detailed discussion of all topics introduced here will be presented by Goulet (1984).

\section{ACKNOWLEDGMENTS}

We would like to thank Jacques Crovisier for providing us with information regarding the Nancay $21 \mathrm{~cm}$ Absorption Survey. We thank NSERC for financial support. 
Crovisier, J., Kazes, J., and Aubry, D. 1978, Astronomy and Astrophysics Suppl., 32, 205 .

Goulet, T., 1984, M.Sc. Thesis, Untversity of British Columbia, (1n preparation).

Helfer, H.L. 1961, Astronomical Journal, 66, 160.

Henderson, A.P. 1973, Astronomical Journal, 78, 381.

Hirshfeld, A., and Sinnott, R. 1982, Sky Catalogue 2000.0, Volume 1,

Cambridge University Press and Sky Publishing Corporation.

McGee, R.X., and Murray, J.D. 1961, Australian Journal of Physics, 14, 260.

Mihalas, D., and Binney, J. 1981, Galactic Astronomy, Structure and

Kinemat1cs, W.H. Freeman, San Francisco.

01 ano, C.A. 1982, Astronomy and Astrophysics, 112, 195.

Takakubo, K. 1967, Bu11. Astr. Inst. Neth., 19, 125.

Weaver, H. 1974, in Highlights of Astronomy, Vol. 3, G. Contopoulos (ed.), D. Reide1 Publishing Co., Dordrecht.

TABLE I

Velocity Field Coefficlents and Velocity Ellipsoid Parameters

$\begin{array}{cccccc}\begin{array}{c}21 \mathrm{~cm} \\ \text { absorption }\end{array} & \begin{array}{c}21 \mathrm{~cm} \\ \text { intercloud }\end{array} & \begin{array}{c}\mathrm{BV} \\ \mathrm{d} 200 \mathrm{pc}\end{array} & \mathrm{BI}-\mathrm{IV} & \mathrm{AV} & \mathrm{K}-\mathrm{G} \text { iants } \\ 281 & 406 & 330 & 451 & 1061 & 981 \\ 395 & 418 & 133 & 692 & 109 & 167\end{array}$

$\langle d\rangle$ pc

Analyzed

Taylor Series

Coefficients

\begin{tabular}{|c|c|}
\hline$K(1)$ & $\mathrm{km} \mathrm{s}^{-1}$ \\
\hline $\begin{array}{l}K(2)=-u_{0} \\
K(3)=-v_{0}^{0} \\
K(4)=-w_{0}^{0}\end{array}$ & $"$ \\
\hline $\begin{array}{l}K(5)=\partial u / \partial x \\
K(6)-\partial v / \partial y \\
K(7)=\partial w / \partial z\end{array}$ & $\begin{array}{c}\mathrm{km} \mathrm{s^{-1 }} \mathrm{kpc}^{-1} \\
"\end{array}$ \\
\hline $\begin{array}{l}K(8)={ }^{\prime} A \\
K(9) \\
K(10)\end{array}$ & ". \\
\hline
\end{tabular}

\begin{tabular}{|c|c|c|c|c|c|c|c|c|}
\hline - & - & \multicolumn{2}{|c|}{$0.7 \pm 1.8$} & $3.4 \pm 0.8$ & \multicolumn{2}{|c|}{$-0.2 \pm 1.0$} & \multicolumn{2}{|c|}{$2.5 \pm 1.6$} \\
\hline $\begin{array}{l}-11.0 \pm 0.8 \\
-16.3 \pm 1.1 \\
-9.5 \pm 1.2\end{array}$ & $\begin{array}{l}-10.1 \pm 0.3 \\
-16.2 \pm 0.3 \\
-11.7 \pm 0.5\end{array}$ & \multicolumn{2}{|c|}{$\begin{array}{r}-9.1 \pm 0.9 \\
-18.2 \pm 1.0 \\
-7.0 \pm 1.5\end{array}$} & $\begin{array}{r}-8.2 \pm 0.9 \\
-17.4 \pm 0.8 \\
-6.7 \pm 2.0\end{array}$ & \multicolumn{2}{|c|}{$\begin{array}{l}-11.6 \pm 1.0 \\
-11.7 \pm 1.3 \\
-6.8 \pm 1.1\end{array}$} & \multicolumn{2}{|c|}{$\begin{array}{l}-10.5 \pm 1.5 \\
-18.6 \pm 1.7 \\
-10.2 \pm 1.7\end{array}$} \\
\hline $\begin{array}{r}8.1 \pm 2.4 \\
-0.2 \pm 3.8 \\
-11.5 \pm 5.9\end{array}$ & $\begin{array}{r}4.2 \pm 0.6 \\
-0.1 \pm 0.9 \\
20.3 \pm 5.4\end{array}$ & $\begin{array}{r}-2 \\
30 \\
24\end{array}$ & $\begin{array}{l} \pm 14 \\
\pm 14 \\
\pm 25\end{array}$ & $\begin{array}{l}-2.8 \pm 1.1 \\
-4.0 \pm 1.2 \\
-6.9 \pm 5.6\end{array}$ & $\begin{array}{r}-15 \\
4 \\
8\end{array}$ & $\begin{array}{l} \pm 11 \\
\pm 12 \\
\pm 15\end{array}$ & $\begin{array}{r}-29 \\
-9 \\
6\end{array}$ & $\begin{array}{l} \pm 13 \\
\pm 13 \\
\pm 15\end{array}$ \\
\hline $\begin{array}{c}(15.0 \pm 2.6) \\
6.9 \pm 4.0 \\
3.1 \pm 2.7\end{array}$ & $\begin{array}{c}(15.0 \pm 0.6) \\
3.3 \pm 1.1 \\
2.3 \pm 0.9\end{array}$ & $\begin{array}{r}12 . \\
-29 \\
15\end{array}$ & $\begin{array}{l}7 \pm 6.8 \\
\pm 12 \\
\pm 11\end{array}$ & $\begin{array}{r}11.2 \pm 0.8 \\
-9.8 \pm 3.4 \\
5.4 \pm 2.4\end{array}$ & $\begin{array}{r}11 . \\
1 . \\
2 .\end{array}$ & $\begin{array}{l}0 \pm 7.4 \\
8 \pm 8.5 \\
7 \pm 8.1\end{array}$ & $\begin{array}{r}4 . \\
16 . \\
0 .\end{array}$ & $\begin{array}{l}9 \pm 7.8 \\
8 \pm 9.3 \\
2 \pm 9.2\end{array}$ \\
\hline$-26^{\circ}$ & $-8^{\circ}$ & & & $-2^{\circ}$ & & $3^{\circ}$ & & $6^{\circ}$ \\
\hline
\end{tabular}

Velocity Ellipsoid

Parameters

$\sigma$
$\left\langle u^{2}\right\rangle^{\frac{1}{2}}$
$\left\langle v^{2}\right\rangle^{\frac{1}{2}}$
$\left.\left\langle w^{2}\right\rangle\right\rangle^{\frac{3}{2}}$
$l_{v}$

$\begin{array}{cc}6.8 & 2.7 \\ - & - \\ - & - \\ - & - \\ - & -\end{array}$

10.3
11.6
9.4
7.7
$20^{\circ}$

11.2
10.6
10.9
14.1

13.2

23.3

$17.4 \quad 27.2$

$11.2 \quad 20.8$

$9.9 \quad 19.9$

$17^{\circ} \quad 23^{\circ}$ 

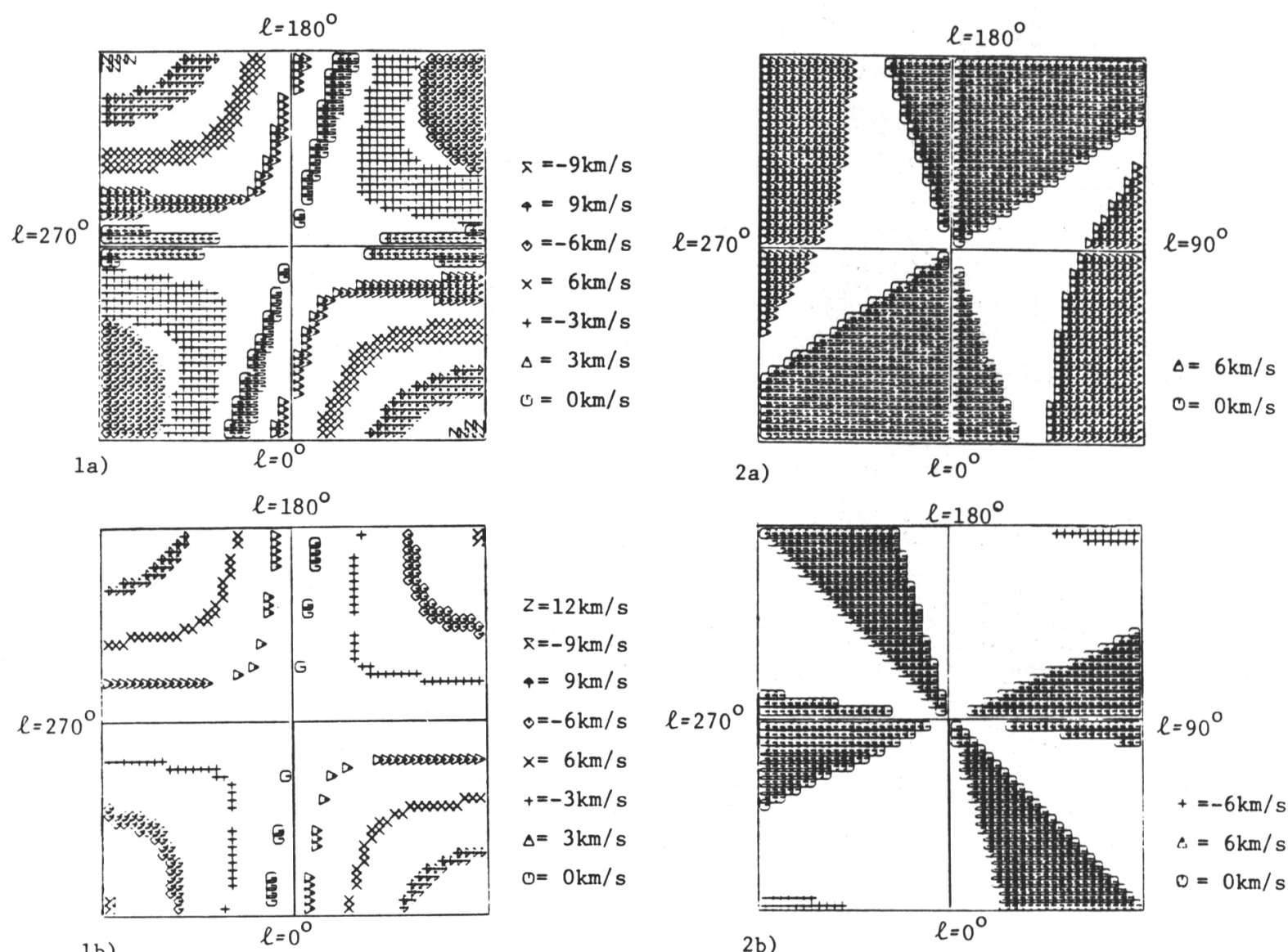

1b)

$\ell=180^{\circ}$

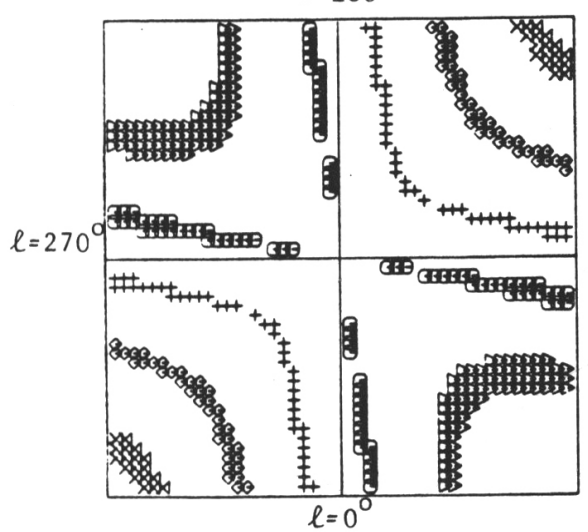

1c)

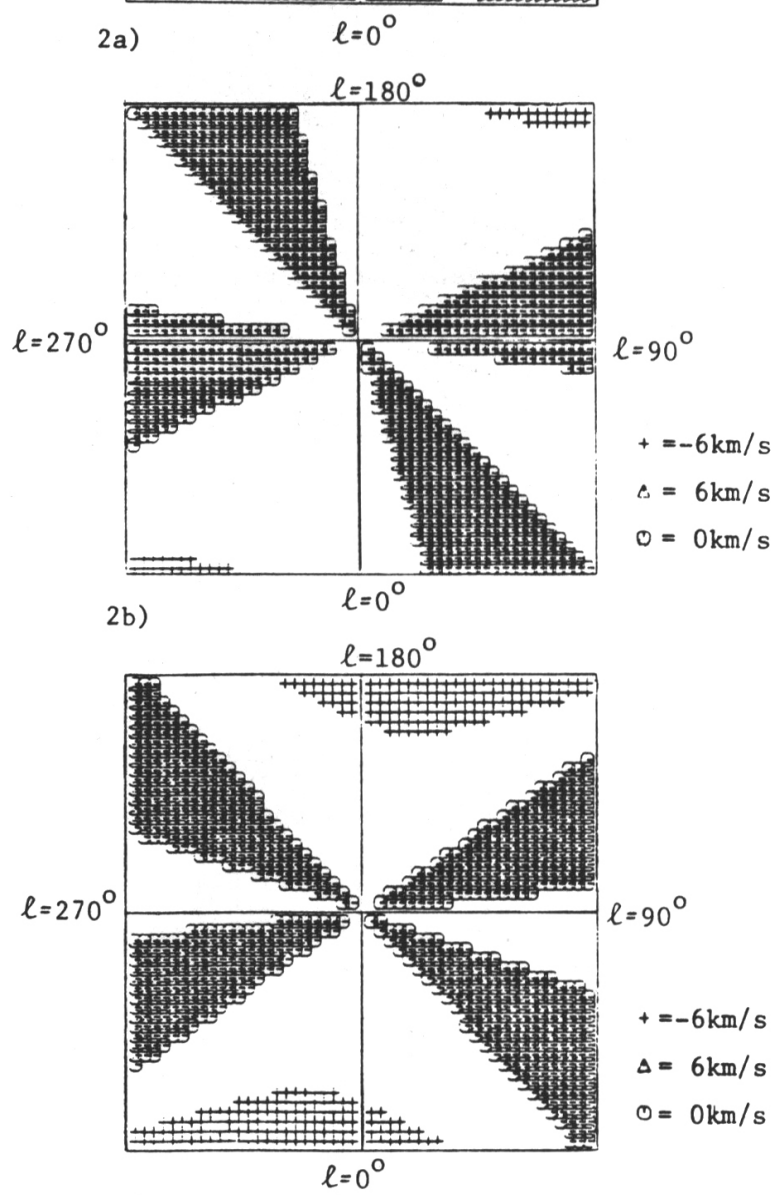

2c)
Figure 1. - Velocity fields in the Galactic midplane ( $x-y$ plane) for (a) $21 \mathrm{~cm}$ absorption, (b) $21 \mathrm{~cm}$ intercloud and (c) BI-IV. Each box has a half-width of $500 \mathrm{pc}$.

.

$\boldsymbol{x}=-9 \mathrm{~km} / \mathrm{s}$

$\uparrow=9 \mathrm{~km} / \mathrm{s}$ $0=-6 \mathrm{~km} / \mathrm{s}$ $x=6 \mathrm{~km} / \mathrm{s}$ $+=-3 \mathrm{~km} / \mathrm{s}$ $\Delta=3 \mathrm{~km} / \mathrm{s}$ $o=0 \mathrm{~km} / \mathrm{s}$ 

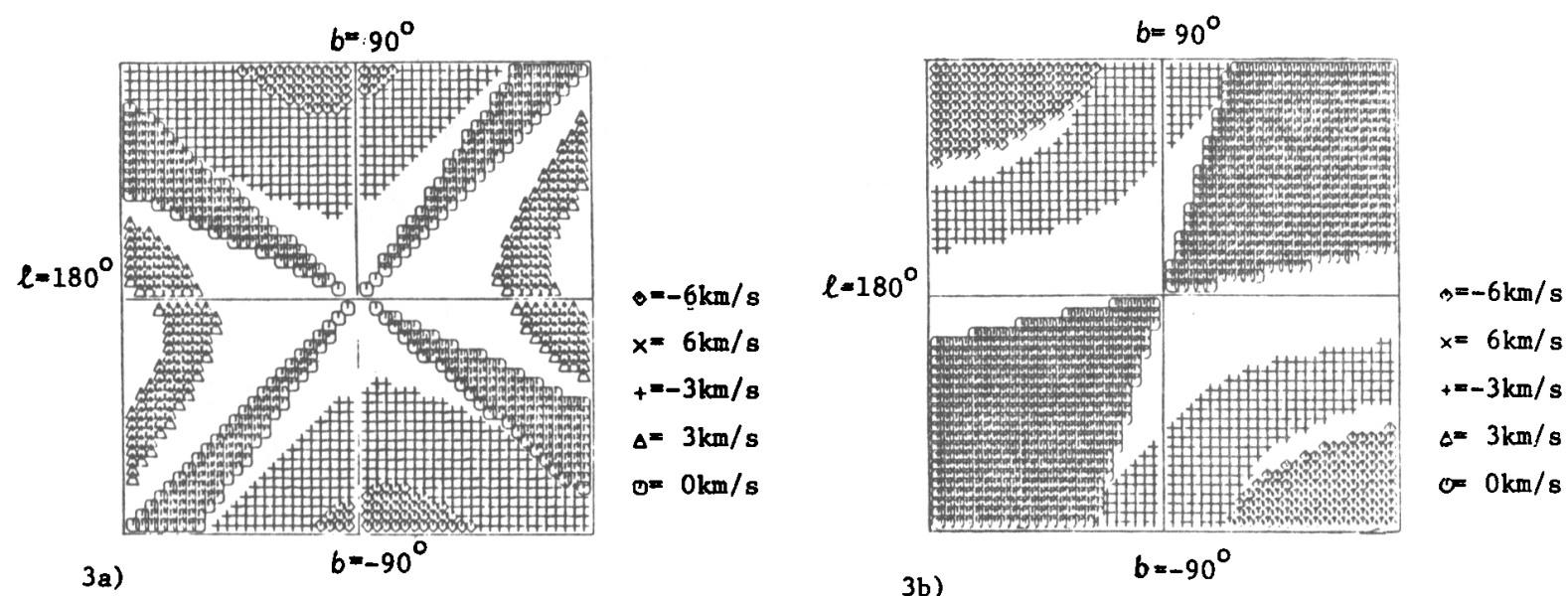

Figure 3. - Velocity fields perpendicular to the Galactic midplane (in the x-z plane) for (a) $21 \mathrm{~cm}$ absorption and (b) BI-IV. Each box has a halfwidth of $500 \mathrm{pc}$. 